\title{
ANÁLISE DO COMPORTAMENTO MECÂNICO DE CONCRETO COM DIFERENTES TIPOS DE ADIÇÃO DE FIBRAS ATRAVÉS DA PROPAGAÇÃO DE ONDAS DE ULTRASSOM
}

\author{
Rodrigo Rogério Cerqueira da Silva ${ }^{1 *}$ \& Luis Henrique de Sampaio Bonfim ${ }^{2}$
}

\begin{abstract}
RESUMO
SILVA, R.R.C. da.; BONFIM, L.H.S. Análise do comportamento mecânico de concreto com diferentes tipos de adição de fibras através da propagação de ondas de ultrassom. Perspectivas Online: Exatas \& Engenharia, v. 09, n. 25, p. 1 - 19, 2019.

A adição de fibras de aço, polipropileno e borracha ao concreto pode alterar propriedades mecânicas como a resistência à flexão, compressão e elasticidade, além de evitar fissuras. Também há alteração nas propriedades físicas, por exemplo, a absorção de temperatura, sons e o bloqueio da umidade. Levando em consideração que atualmente a avaliação da resistência de estruturas seja realizada através de métodos destrutivos, o ensaio de ultrassom permite avaliar a integridade dos materiais através de pulsos ultrassônicos. A análise conjunta dos dados de ultrassom com dados de ensaios destrutivos permite a confiabilidade dos dados. O objetivo da

presente pesquisa foi analisar amostras de concreto convencional e com $2 \%$ de adição de diferentes tipos de fibras (aço, borracha e polipropileno). Com intenção de comparar a interferência das fibras nas velocidades de propagação de ondas $(\mathrm{V})$, para as frequências de $45 \mathrm{kHz}$ e $80 \mathrm{kHz}$ e nas propriedades mecânicas do concreto através dos ensaios de resistência à compressão (fc) e módulo de elasticidade (Eci). Foram realizadas correlações entre os parâmetros obtidos por ensaios destrutivos e propagação de ondas, demonstrando que o ensaio de ultrassom é capaz de inferir na qualidade de peças de concreto.
\end{abstract}

Palavras-chave: ensaio de ultrassom; resistência à compressão, resistência à tração por compressão diametral, módulo de elasticidade, concreto com fibras. 


\begin{abstract}
The addition of steel fibers, polypropylene and rubber to concrete can change mechanical properties such as flexural strength, compression and elasticity, as well as preventing cracking. There are also changes in physical properties, such as temperature absorption, sounds and moisture blockage. Considering that currently the evaluation of the strength of structures is performed by destructive methods, the ultrasound test allows to evaluate the integrity of materials through ultrasonic pulses. Combined analysis of ultrasound data with destructive test data enables data reliability. The objective of

this research was to analyze samples of conventional concrete with $2 \%$ addition of different types of fibers (steel, rubber and polypropylene). With the intention of comparing the interference of the fibers in the wave propagation velocities $(\mathrm{V})$, in the frequencies of $45 \mathrm{kHz}$ and $80 \mathrm{kHz}$ and in the mechanical properties of the concrete through the tests of compressive strength (fc) and modulus of elasticity (Eci). Correlations between the parameters obtained by destructive tests and wave propagation were performed, demonstrating that the ultrasound test is able to infer the quality of concrete pieces.
\end{abstract}

Keywords: ultrasound test; compressive strength; tensile strength in diametral compression, modulus of elasticity, concrete with fibers.

\footnotetext{
${ }^{1}$ Universidade Nove de Julho - UNINOVE - Departamento de Ciências Exatas - Rua Guaranésia, 425, Vila Maria, SP, CEP: 02112-000, Brasil;

(*) e-mail: rodrigorogerio@uni9.pro.br

Data de recebimento: 22/06/19. Aceito para publicação: 08/08/19.
} 


\section{INTRODUÇÃO}

O mercado da construção civil encontra-se em constante evolução e a utilização de materiais alternativos na composição do concreto está tornando-se cada vez mais frequente. A adição de fibras ao concreto surge como uma boa alternativa para controle das patologias. Estudos realizados por Figueiredo (1992), Akasaki (2001), Bentur e Mindess (2007), Battagin (2007), Chun et al. (2007), Goes et al. (2016), Oliveira (2014), Lucena (2017), Ehrenbring et al. (2018) e Herscovic et al. (2019), relatam que uma das características atribuídas das fibras é o aumento da ductilidade do concreto, reduzindo o comportamento frágil em relação a trincas e fissuras, além de proporcionar melhorias na absorção de impactos em zonas de fissuração.

Fibras podem modificar tanto as características mecânicas do concreto, tornando-o mais resistente à flexão, compressão e elasticidade, como características físicas, absorvendo temperatura, sons e não propagando umidade. Segundo Figueiredo (1992), a utilização de fibras no concreto projetado auxilia no ganho de resistência à compressão e melhora o módulo de elasticidade com resultados iguais ou superiores ao concreto com utilização de telas. No entanto, Bentur e Mindess (2007) afirmam que as fibras possuem relação direta com o ganho de resistência à tração e aumentam a capacidade de deformação, além de atuarem no controle das fissuras e distribuição equilibrada de tensões, mas com pouco ou nenhum efeito no aumento da resistência à compressão e módulo de elasticidade.

De acordo com Metha e Monteiro (2008), o tipo de fibra mais utilizado para fim estrutural e não estrutural ainda é a de aço. Na última década, as fibras vêm sendo objeto de estudo para ser empregadas nas mais diversas aplicações do concreto.

Pesquisadores avaliaram fibras de aço (Balendran et al., 2002; Lau e Anso, 2006; Figueiredo, 2011; Domagala, 2011), verificando melhorias quanto ao seu módulo de elasticidade e sua resistência à compressão axial. Estudos realizados com fibras de polipropileno por (Richardson, 2006; Gencel, 2011; Lucena, 2017; Rosa Filho et al., 2017) obtiveram aumento na resistência à traçao e redução na quantidade de fissuras quando comparados ao concreto simples. Através da adição de fibras de borracha no concreto (Segre, 1999; Segre et al., 2002; Chun, 2007; Akasaki, 2001; Albuquerque et al., 2002, 2004, 2005; Albuquerque 2009), verificaram que a adição não causa modificaçoes significativas nas resistências mecâncias, porém contribui para que as tensões se distribuíssem de maneira equilibrada, controlando a fissuração.

Fibras de aço e polipropileno atuam de maneira semelhante e podem ser combinadas para que haja um melhor desempenho do concreto, comumente empregadas em peças prémoldadas como segmentos de anéis de túneis e tubos de concreto para galerias. Ambas possuem boa aderência à pasta de cimento devido ao formato corrugado (na maioria das aplicações), porém, a fibra de aço por ser menos maleável, alcança melhores resultados no controle das fissuras provenientes da compressão. A borracha pode ser utilizada em formato de grãos ou fibras em pisos industriais, pavimentação e New Jersey, podendo substituir parcialmente o agregado graúdo, deixando o concreto mais leve, porém menos resistente em decorrência da baixa aderência à pasta de cimento. 
De acordo com Meta e Monteiro (2008) os tipos de fibras e sua fração interferem diretamente nas propriedades do concreto e para uma proporção de $50 \mathrm{Kg}$ de cimento a adição de fibras menor que $1 \%$, atua com o objetivo apenas de reduzir a fissuração da peça. Adições de fibra entre $1 \%$ e $2 \%$, elevam de forma considerável o módulo de ruptura, dureza e resistência ao impacto da peça. Para adição de valores superioes à $2 \%$, há endurecimento da peça por deformação dos compósitos e aumento do índice de vazios, o que reduziria a resistência do concreto.

Uma das razões para a falta de controle tecnológico na construção civil está relacionada à necessidade de recorrer à estrutura laboratorial de ensaios destrutivos, com custos elevados, além de comprometer as estruturas analisadas.. A técnica de avaliação das estruturas por meio da utilização de ensaios não destrutivos vem crescendo continuamente em todo o mundo, abrangendo vários ensaios. Dentre os ensaios que se destacam pela praticidade, custo-benefício, portabilidade e facilidade no manuseio, está o ultrassom. No entanto, é necessário adequar o ensaio à situação específica ("in loco") e às características do material, e assim obter parâmetros e metodologia que permitam confiabilidade em sua aplicação.

Estudos realizados por Sturrup et al. (1984), Ohdaira e Masuzawa (2000), Nogueira e Willam (2001), Popovics (2001), Evangelista (2002), Mohammed e Hasan (2016), Silva (2017) e Yu et al. (2019), mostraram que o tipo de agregado, cimento e a relação de água e cimento, idade do concreto, condições de cura, granulometria dos agregados, influenciam npropagação de ondas. Em relação as fibras, Pillar (2014) e Bosco (2015) mostraram que as velocidades de ultrassom sofrem variações em relações ao teor e tipos de fibras incorporados nas misturas de concretos.

O presente trabalho tem como objetivo analisar o comportamento das fibras de aço, borracha e polipropileno adicionadas em concreto a fim de definir as vantagens e desvantagens da adição destas, através da avaliação das propriedades de resistência à compressão axial, diametral, módulo de elasticidade e ensaio de ultrassom.

\section{MATERIAS E MÉTODOS}

\subsection{Amostragem}

A amostragem foi composta no total de 48 corpos de prova de $100 \mathrm{~mm}$ de diâmetro e $200 \mathrm{~mm}$ de altura, em conformidade com a norma ABNT NBR 5738, 2015), confeccionando 12 repetições para quatro traços com as seguintes características: sem adição de fibras (CPS), fibras aço (CPFA), fibras de polipropileno (CPFP) e fibras de borracha (CPFB), considerando a mesma proporção de agregados (graúdos e miúdos) e relação a/c (água/cimemto)) A variação da amostragem com e sem fibra permitirá obter a contribuição mecânica ao concreto através do desempenho de cada tipo de fibra.

\subsection{Preparação e características do concreto dos corpos de prova}

Para confecção dos traços foram utilizadas água potável, cimento Portland do tipo CP II-Z-32, comumente utilizado em elementos estruturais, agregado miúdo natural quartzoso (areia média lavada), fibra (aço, polipropileno e borracha) conforme Figura 1, e agregado graúdo britado (pedrisco) mineralogia tipo granito. O agregado graúdo britado foi escolhido

Persp. Online: exatas \& eng., Campos dos Goytacazes, 25 (09) 01 - 19 - 2019

seer.perspectivasonline.com.br 
devido ao fato de ser um dos mais abundantes do Brasil. O traço básico definido possuí as proporções entre os materiais de 1:2,5:3,5 (cimento, areia, pedrisco) sendo o cimento em massa, e os agregados em volume, com adição das fibras com porcentagem correspondente a $2 \%$ do peso do cimento e relação água/cimento $(0,7)$.

As fibras acrescentadas ao concreto possuem comprimentos entre 4 e $6 \mathrm{~cm}$, sendo as fibras de borracha mais espessas e alongadas, obtidas através do processo de frisagem de pneus, o que as deixam com um comprimento irregular, porém foram cortadas para possuir um tamanho próximo das demais. Não foi utilizado nenhum tipo de aditivo durante a confecção dos traços experimentais, sendo que, após a confecção os corpos de prova de concreto, os mesmos foram curados ao ar livre, protegidos contra ações climáticas, e foram desmoldados após 24 horas e colocados em câmera úmida até 28 dias.

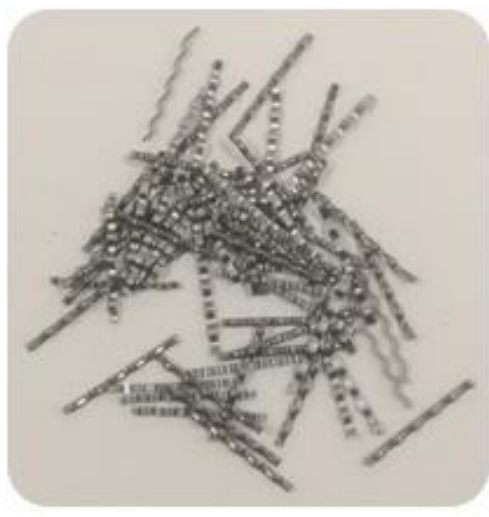

(a)

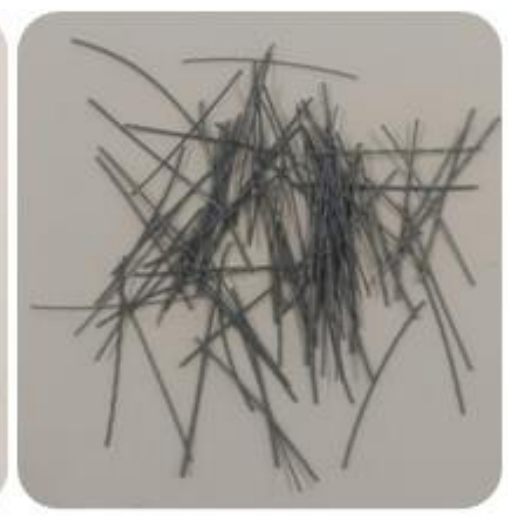

(b)

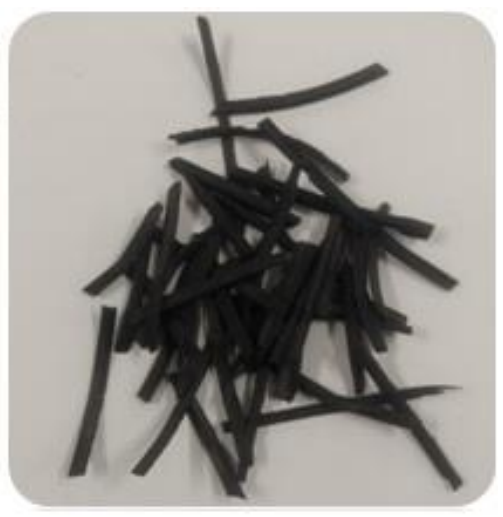

(c)

Figura 1: Fibras adicionadas ao concreto: (a) aço, (b) polipropileno, (c) borracha.

\subsection{Slump e Densidade}

Logo após a produção do concreto executaram-se os ensaios slump realizados conforme NM 67 (1998). Após 28 dias, a massa de cada corpo de prova foi determinada por pesagem em balança de precisão com resolução de $0,1 \mathrm{~g}$ e, para cálculo do volume e densidade da amostras, as dimensões foram determinadas com o auxílio do paquímetro digital.

\subsection{Ensaio de propagação de onda}

Após o período de cura, as amostras foram submetidas ao ensaio de propagação de ondas utilizando equipamento de ultrassom (USLAB, Agricef, Brasil) e transdutores longitudinais de faces planas e frequências 45 e $80 \mathrm{KHz}$ (Figura 2a). Antes da realização dos ensaios, o equipamento foi calibrado utilizando-se um material acrílico cuja velocidade é conhecida.

O ensaio de ultrassom foi realizado com 3 repetições para cada corpo-de-prova, sendo uma ao centro e duas nas extremidades, determinando-se, desta forma, o tempo médio de percurso da onda ( $\left.\mathrm{t}_{\text {médio }}\right)$ em cada um deles. A variação da frequência dos transdutores de 45 
$\mathrm{KHz}$ para $80 \mathrm{KHz}$ tem como objetivo avaliar a diferença entre os comprimentos de ondas $(\lambda)$ na detecção de anomalias dentro da amostra. De acordo com Bucur (2006), para evitar excessivas atenuações, as dimensões do comprimento de onda não podem ser menores do que a dimensão dos elementos atômicos do material estudado.

O ensaio direto foi realizado posicionando os transdutores em faces opostas do corpo de prova (Figura 2b), conforme proposto pela norma brasileira (NBR 8802, 2013). Durante os ensaios foi utilizado como acoplante gel medicinal nas faces dos transdutores, para garantir a perfeita propagação do som através do corpo de prova, e evitar perdas de sinais por impedância entre os transdutores e os corpos de prova. A partir do comprimento do corpo de prova (L) e do resultado do tempo de propagação das ondas (T), calculou-se a velocidade de propagação das ondas de ultrassom (V) equação 1 conforme (NBR 8802, 2013). De posse da velocidade $(\mathrm{V})$, densidade do corpo de prova $(\rho)$ e coeficiente de poisson $(v)$, de acordo com a norma britânica (BS 1881: Part 203, 1986) é possível obter o módulo de elasticidade dinâmico (Ed), conforme equação 2.

$$
\begin{gathered}
V=\frac{L}{T} \\
E d=\rho \cdot V^{2} \cdot \frac{(1+v) \cdot(1+2 v)}{1-v}
\end{gathered}
$$

Onde:

fc $=$ resistência à compressão $(\mathrm{Mpa})$;

$\mathrm{V}=$ Velocidade de propagação de ondas $\left(\mathrm{m} \cdot \mathrm{s}^{-1}\right)$;

$\mathrm{T}=$ tempo $(\mathrm{s}) ; \rho=$ densidade do concreto $\left(\mathrm{kg} \cdot \mathrm{m}^{-3}\right)$;

$\mathrm{Ed}=$ módulo de elasticidade dinâmico $(\mathrm{GPa})$.

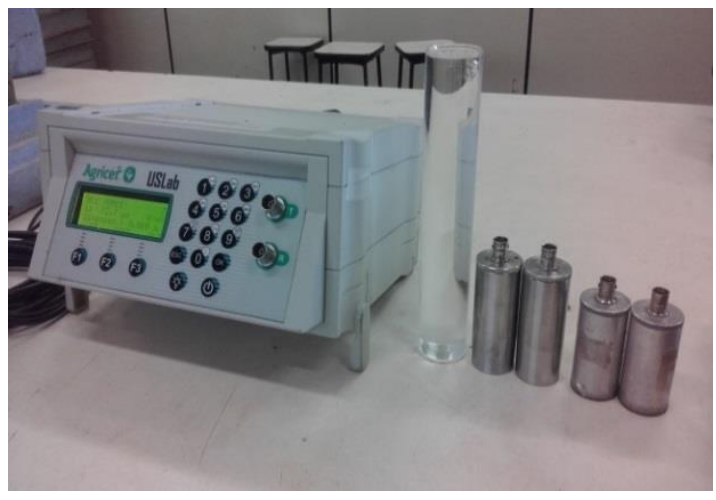

(a)

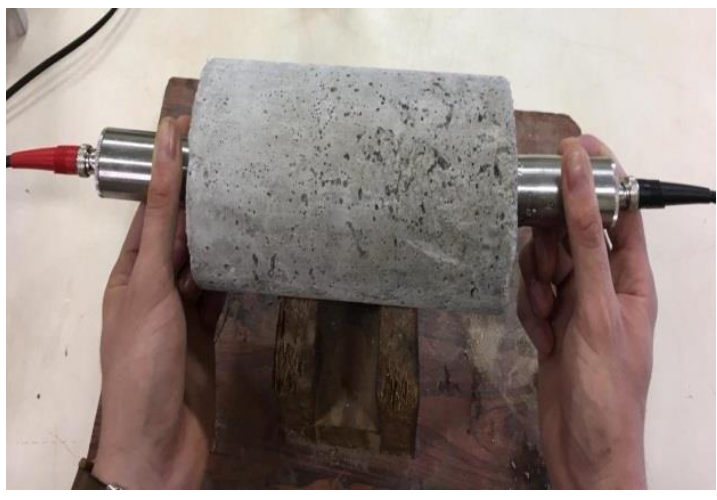

(b)

Figura 2: (a) Aparelho de ultrassom USLAB, acrílico de calibração, transdutores com frequência de 45 e $8 \mathrm{KHz}$, (b) ensaio de ultrassom em corpo de prova.

\subsection{Ensaios estáticos de compressão}

Os ensaios foram realizados em máquina hidráulica, seguindo as especificações de acordo norma brasileira (NBR 5739, 2018), sendo realizado para cada 6 amostras ensaio de resistência à compressão (fc) calculado de acordo com a equação 3 (NBR 5739, 2018), ensaio 
resistência à tração por compressão diametral e cálculo (ft,D) - equação 4 (NBR 7222, 2011), e a resistência característica à compressão do concreto (fck) calculado pela equação 5 , sendo o desvio padrão (s) utilizado igual a 4,0 MPa, devido aos materiais serem dosados em massa e a água de amassamento ser corrigida em função da correção da umidade dos agregados. Os corpos de prova foram instrumentados com extensômetros de resolução de $0,01 \mathrm{~mm}$, para a determinação do módulo de elasticidade (Eci) calculado de acordo com a norma brasileira (NBR 8522, 2017) equação 6.

$$
\begin{gathered}
f c=\frac{4 \cdot F}{\pi \cdot D^{2}} \\
f t, D=\frac{2 \cdot F}{D \cdot L} \\
f c k=f c m-1,65 . s \\
E_{c i}=\frac{\sigma_{b}-0,5}{\varepsilon_{b}-\varepsilon_{a}}
\end{gathered}
$$

Onde:

fc $=$ Resistência à compressão do corpo de prova $(\mathrm{MPa})$;

$\mathrm{F}=$ força máxima alcançada $(\mathrm{N})$;

$\mathrm{ft}, \mathrm{D}=$ resistência à tração por compressão diametral e cálculo;

$\mathrm{D}=$ diâmetro do corpo de prova $(\mathrm{mm})$;

$\mathrm{L}=$ comprimento do corpo de prova $(\mathrm{mm})$;

fck $=$ Resistência característica do concreto à compressão (MPa);

$\mathrm{fcm}=$ Média aritmética dos valores de fc $(\mathrm{MPa})$;

$\mathrm{s}=$ Desvio padrão $(\mathrm{MPa})$.

$\sigma_{\mathrm{b}}=$ tensão $(\mathrm{MPa})$ obtida com $30 \%$ da resistência à compressão;

$0,5=$ valor da tensão de referência inicial $(\mathrm{MPa})$;

$\varepsilon_{\mathrm{b}}$ e $\varepsilon_{\mathrm{a}}=$ deformações específicas do concreto sob a tensão correspondente a $30 \%$ da resistência à compressão e sob a tensão de referência inicial, respectivamente.

\section{RESULTADOS E DISCUSSÃO}

\subsection{Slump e Densidade}

Os valores de Slump e as densidades médias dos concretos produzidos variaram de acordo com os diferentes tipos de fibras, conforme o esperado devido a suas densidades (Tabela 1). Apesar das variações, os valores de todas as densidades estão dentro dos limites considerados normais para o concreto, de $2000 \mathrm{~kg} \cdot \mathrm{m}^{-3}$ a $2800 \mathrm{~kg} \cdot \mathrm{m}^{-3}$, segundo norma brasileira (ABNT NBR 9778, 2015). Apesar do fator a/c (0,7) o slump das amostras realizados conforme (NM 67, 1998) não foi baixo devido à correção da umidade da areia (NBR 16097:12), para que a água adicionada não prejudicasse o traço estipulado. Foi determinado um teor de umidade no agregado de aproximadamente $20 \%$ e essa quantidade teve de ser retirada da água que seria acrescentada durante a mistura do concreto. Outro fator relacionado a diminuição da trabalhabilidade do concreto está relacionado a adição das fibras, 
sendo que durante a mistura contribuíram para se tornar mais consistente.

De acordo com Cimolin e Godinho (2015), é comum que haja perda de abatimento quando se adiciona fibras ao concreto, pois elas causam um "travamento" entre os componentes da mistura, conforme indicado por o que pode variar de acordo com a quantidade de fibras que é inserida no concreto.

Tabela 1: Valores de slump e de densidade média dos concretos produzidos com agregados de diferentes origens mineralógicas e fatores água-cimento.

\begin{tabular}{l|c|c}
\hline \multicolumn{1}{c|}{ Tipo de fibra } & Slump $(\mathrm{mm})$ & Densidade média $\left(\mathrm{kg} \cdot \mathrm{m}^{-3}\right)$ \\
\hline CPS - Sem fibra & $10 \pm 2$ & 2617 \\
\hline CPFA - Fibra de aço & $14 \pm 2$ & 2619 \\
\hline CPFB - Fibra de borracha & $12 \pm 2$ & 2602 \\
\hline CPFP - Fibra de polipropileno & $11 \pm 2$ & 2637 \\
\hline
\end{tabular}

\subsection{Ensaios de Ultrassom}

A Tabela 2 apresenta estes resultados entre as amostras de concreto convencional e com adição de fibras, através de propagação de ondas de ultrassom.

As diferentes posições das fibras localizadas na composição do concreto em decorrência da sua quantidade de $2 \%$ para cada $50 \mathrm{~kg}$ de cimento e densidade obtiveram efeito insignificante nos tempos de percurso medidos dos pulsos ultrassônico, reduzindo em média a velocidade em torno de 3\% da amostra sem adição de fibras. De acordo com Acebes et al. (2011), o aumento da porcentagem das fibras de aço causam uma diminuição na velocidade de ultrassom devido à geometria das inclusões de aço.

Tabela 2: Valores médios de velocidade, comprimento de ondas, e número ondas para frequência de 45 e $80 \mathrm{kHz}$.

\begin{tabular}{c|c|c|c|c|c|c|c|c}
\hline \multirow{2}{*}{ Fibra } & \multicolumn{2}{|c|}{ Velocidade } & \multicolumn{2}{c|}{ Ed } & \multicolumn{2}{c|}{ Comprimento } & \multicolumn{2}{c}{$\mathrm{N}^{\mathrm{o}}$ de ondas } \\
\cline { 2 - 9 } & $\begin{array}{c}45 \mathrm{kHz} \\
(\mathrm{m} / \mathrm{s})\end{array}$ & $\begin{array}{c}80 \mathrm{kHz} \\
(\mathrm{m} / \mathrm{s})\end{array}$ & $\begin{array}{c}45 \mathrm{kHz} \\
(\mathrm{m} / \mathrm{s})\end{array}$ & $\begin{array}{c}80 \mathrm{kHz} \\
(\mathrm{m} / \mathrm{s})\end{array}$ & $\begin{array}{c}45 \mathrm{kHz} \\
(\lambda-\mathrm{mm})\end{array}$ & $\begin{array}{c}80 \mathrm{kHz} \\
(\lambda-\mathrm{mm})\end{array}$ & $\begin{array}{c}45 \\
\mathrm{kHz}\end{array}$ & $\begin{array}{c}80 \\
\mathrm{kHz}\end{array}$ \\
\hline Sem fibras & $\begin{array}{c}3477 \\
(2,8)\end{array}$ & $\begin{array}{c}3493 \\
(2,1)\end{array}$ & $\begin{array}{c}28,5 \\
(5,6)\end{array}$ & $\begin{array}{c}28,7 \\
(4,3)\end{array}$ & 77,3 & 43,3 & 2,5 & 4,5 \\
\hline Aço & $\begin{array}{c}3390 \\
(2,4)\end{array}$ & $\begin{array}{c}3470 \\
(1,7)\end{array}$ & $\begin{array}{c}27,2 \\
(5,0)\end{array}$ & $\begin{array}{c}28,5 \\
(4,0)\end{array}$ & 75,3 & 43,8 & 2,6 & 4,5 \\
\hline Borracha & $\begin{array}{c}3375 \\
(1,25)\end{array}$ & $\begin{array}{c}3434 \\
(2,9)\end{array}$ & $\begin{array}{c}26,7 \\
(2,0)\end{array}$ & $\begin{array}{c}27,6 \\
(6,5)\end{array}$ & 75,3 & 43,2 & 2,6 & 4,6 \\
\hline Polipropileno & $\begin{array}{c}3325 \\
(1,55)\end{array}$ & $\begin{array}{c}3369 \\
(2,9)\end{array}$ & $\begin{array}{c}26,2 \\
(3,3)\end{array}$ & $\begin{array}{c}27,0 \\
(6,3)\end{array}$ & 73,9 & 42,2 & 2,7 & 4,7 \\
\hline
\end{tabular}

Onde: Valores em parênteses representam o coeficiente de variação das amostras.

Em virtude da baixa densidade das fibras de polipropileno e borracha, durante a confecção dos traços de concreto utilizou-se maior quantidade de fibras, ocasionando maior 
redução de velocidade de propagação de onda, quando comparadas as fibras de aço, devido à heterogeneidade em sua distribuição na composição. De acordo com Figueiredo (2011), a ausência de homogeneidade na distribuição das fibras e do percentual em cada corpo de prova, gera o efeito de ouriços fenômeno causado pela aglomeração e embolamento das fibras que está diretamente relacionado ao tamanho e processo durante a mistura. Através de software estatístico (Statgraphic) foi possível avaliar através dos modelos two sample comparison para prever se existe diferença significante entre os valores de velocidade entre as amostras considerando os diferentes tipos de fibras e frequência dos transdutores, conforme apresentado na Tabela 3.

Tabela 3: Valores estatísticos das velocidades para as amostras com fibras de aço, borracha e polipropileno.

\begin{tabular}{c|c|c}
\hline Dados estatísticos & Frequência $45 \mathrm{KHz}$ & Frequência $80 \mathrm{KHz}$ \\
\hline Amostras avaliadas & 48 & 48 \\
\hline Média das velocidades & 3392 & 3442 \\
\hline Desvio padrão & 62,95 & 54,05 \\
\hline Coeficiente de variação & $1,86 \%$ & $1,57 \%$ \\
\hline Mínimo & 3325,33 & 3369,23 \\
\hline Máximo & 3476,64 & 3493,21 \\
\hline Assimetria & 0,67673 & $-0,763256$ \\
\hline Curtose & 0,668703 & 0,114098 \\
\hline
\end{tabular}

Um dos dados de maior interesse na avaliação das amostras são a assimetria e curtose padronizada, que podem ser usadas para determinar se as amostras correspondem a uma distribuição normal. Valores das estatísticas fora do intervalo de -2 a +2 indicam desvios significativos da normalidade, o que tenderia a invalidar os procedimentos estatísticos aplicados a verificação dos dados. Nesse caso, os dois valores de assimetria e curtose padronizados estão dentro do intervalo esperado, indicando que as velocidades das amostras são estatisticamente significativas.

Os coeficientes de variação estão dentro dos padrões de aceitação de acordo com a ASTM C 597 (2002), que determina que a variação dos resultados dos testes deverá estar dentro de até $2 \%$, para ensaios de ultrassom realizados em amostras de concreto. A comparação das médias das velocidades das amostras para ambas a s frequências (CPS, CPFA, CPFB, CPFP), através da análise de variância pelo método ANOVA indicam com diferença significativa para $\mathrm{P}$-valor menor que 0,05 . Como o intervalo das amostras possui Pvalor $=0,28$, não há diferença estatisticamente significante entre as médias das velocidades das amostras considerando as duas frequências e diferentes tipos de fibras, apresentando um nível de confiança de $95,0 \%$.

\subsection{Ensaios Destrutivos}

A Tabela 4 apresenta a resistência característica (fck), resultados médios da resistência à compressão (fc), resistência à tração por compressão diametral (ft,D) e módulo de elasticidade (Eci) das amostras sem adição e com adição de fibras. 
Tabela 4: Valores médios (fck), (fc), (ft,D) e (Eci) pra corpos de prova sem adição de fibras (CPSF), com adição de fibras de aço (CPFA), fibras de borracha (CPFB) e fibras de polipropileno (CPFP).

\begin{tabular}{c|c|c|c|c}
\hline Amostra & $\begin{array}{c}\mathrm{fck} \\
(\mathrm{MPa})\end{array}$ & $\begin{array}{c}\mathrm{fc} \\
(\mathrm{MPa})\end{array}$ & $\begin{array}{c}\mathrm{ft}, \mathrm{D} \\
(\mathrm{MPa})\end{array}$ & $\begin{array}{c}\mathrm{Eci} \\
(\mathrm{GPa})\end{array}$ \\
\hline \multirow{2}{*}{$\mathrm{CPSF}$} & 12 & $\begin{array}{c}18,7 \\
(12)\end{array}$ & $\begin{array}{c}6,85 \\
(8,6)\end{array}$ & $\begin{array}{c}21,80 \\
(4,8)\end{array}$ \\
\hline \multirow{2}{*}{$\mathrm{CPFA}$} & 13 & $\begin{array}{c}19,6 \\
(12)\end{array}$ & $\begin{array}{c}8,6 \\
(14,9)\end{array}$ & $\begin{array}{c}22,30 \\
(14)\end{array}$ \\
\hline CPFB & 10 & $\begin{array}{c}16,5 \\
(7)\end{array}$ & $\begin{array}{c}6,6 \\
(14)\end{array}$ & $\begin{array}{c}20,44 \\
(14,3)\end{array}$ \\
\hline CPFP & 11 & $\begin{array}{c}17,4 \\
(11,5)\end{array}$ & $\begin{array}{c}6,9 \\
(6,5)\end{array}$ & $\begin{array}{c}21,02 \\
(9,4)\end{array}$ \\
\hline
\end{tabular}

Onde: Valores em parênteses representam o coeficiente de variação das amostras.

Os resultados do ensaio de resistência à compressão e tração diametral, pela análise de médias, indica que a adição do teor de fibras na mistura não obteve influencia na resistência à compressão para as fibras de polipropileno e borracha em relação as amostras sem adição de fibras.

A baixa densidade do polipropileno proporciona aumento em sua quantidade na confecção dos traços, fazendo com que se dispersem e incorpore ar durante o processo de moldagem gerando a perda de resistência. Porém, as fibras de polipropileno atuou de maneira mais efetiva na distribuição das tensões e junção dos agregados após os ensaios mecânicos em relação as amostras CPSF, CPFA e CPFB.

De acordo com estudos realizados por Segre (1999) a borracha de pneu é um material de pouca aderência à matriz do concreto, de forma que não crie ligações com a pasta de cimento, então, onde a borracha se encontra, pode-se considerar como vazios dentro da peça de concreto e isso afeta diretamente a resistência mecânica. As fibras de borracha possuem uma baixa resistência e característica elástica, ou seja, há uma baixa deformação seguida de um rápido rompimento. Seu formato não beneficia, pois não há uma boa aderência a matriz de concreto e seu material não agrega resistência, resultando em um material enfraquecido nos pontos onde a borracha se encontra, rompendo as amostras com baixos escorregamentos não contribuindo para diminuir a fissuração.

No traço com adição de fibras de aço houve um pequeno aumento de aproximadamente 5\%, embora a quantidade seja pequena por conta do seu peso específico, trata-se de um material com alto módulo de elasticidade em relação aos demais, melhorando características como tenacidade e resistência ao impacto.

De acordo com Bentur e Mindess (2007) e Medeiros (2012), pra concretos com teor de fibras menores que $2 \%$, o comportamento da resistência à compressão e módulo de elasticidade, não são alterados quanto o comportamento a tração, não passando de $25 \%$ em relação a amostras sem adição de fibras. Quanto ao módulo de elasticidade, notou-se que houve uma diferença baixa em relação ao traço sem fibras, sendo que o traço com adição de borracha ainda obteve um desempenho inferior aos demais traços estudados devido a sua 
baixa aderência com a mistura do concreto, além de menor resistência à tração, sendo uma das principais causas de perda da resistência e módulo de elasticidade da adição de borracha.

De acordo com Bentur e Mindess (1990) fibras de aço possuem módulo de elasticidade 20 vezes maior que as fibras polipropileno, e resistência à tração 2 vezes maior. Esses tipos de fibras proporcionam um maior travamento dos componentes constituintes do concreto. Segundo Figueiredo (2011), as fibras de aço possuem elevado módulo de elasticidade, proporcionando maior nível de reforço da matriz, mesmo com baixo consumo de fibras.

A Figura 3 indica os tipos de rupturas para cada tipo de fibras. No caso de concreto sem adição de fibras e com adição de fibras de aço, quando as tensões aplicadas superam a resistência da matriz de concreto,ocorreu o fenômeno de ruptura brusca. Foi observado que as fibras de borracha e polipropileno contribuiram para a diminuição da fissuração das amostras. Segundo Medeiros (2012), as fibras se comportam como inclusões inativas, produzindo melhorias periféricas das propriedades mecânicas.

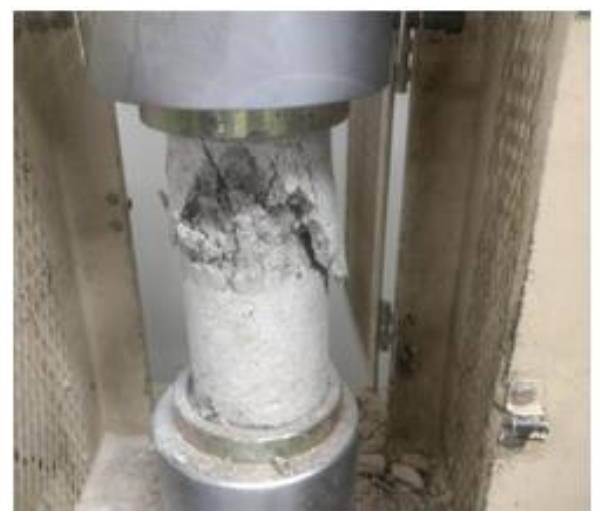

(a)

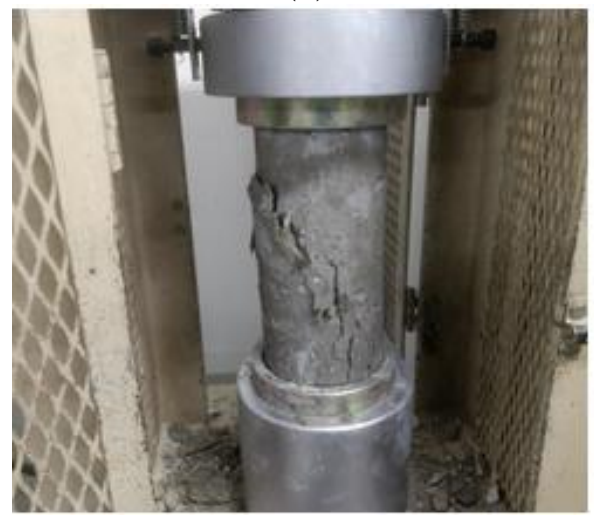

(c)

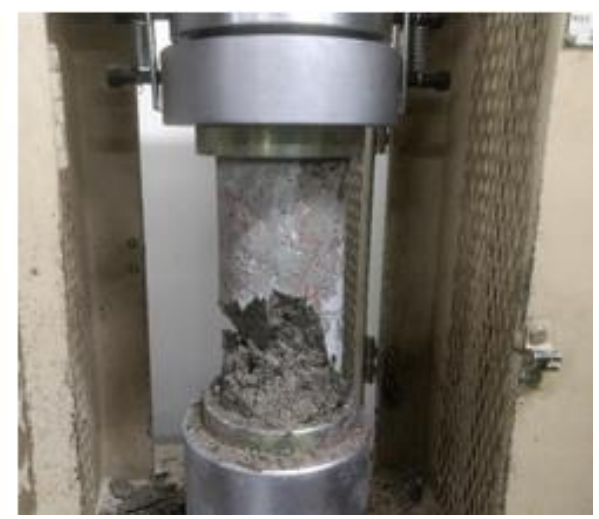

(b)

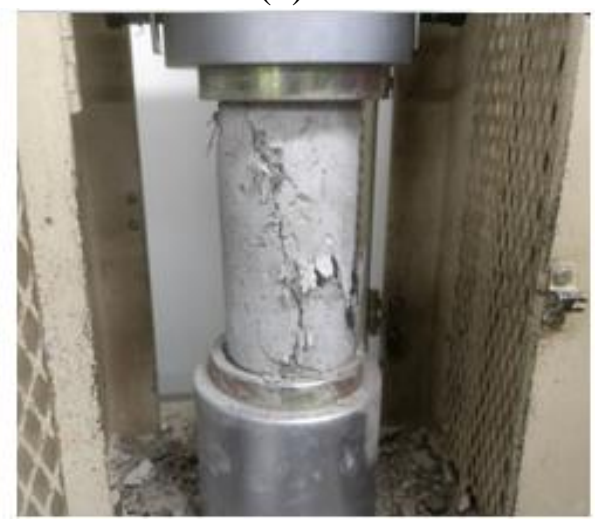

(d)

Figura 3: Tipos de ruptura dos corpos de prova (a) sem fibras -CPSF, (b) fibras de aço CPFA, (c) fibras de borracha - CPFB, (d) fibras de polipropileno - CPFP. 


\subsection{Correlações entre ensaios destrutivos e não destrutivos}

A partir dos parâmetros obtidos por ensaios destrutivos (fc), (ft,D) e (Eci) e por propagação de ondas $(\mathrm{V})$ e (Ed) considerando os 48 corpos de provas, foram realizadas correlações dos resultados experimentais através do software estatístico (Stratgraphics Centurion) para as amostras para cada tipo de adição de fibra. As correlações obtidas entre os ensaios possibilita tornar de forma mais expedita e imediata o comportamento de estruturas e amostras de concreto com adição de fibras através da propagação de ondas. A Tabela 5 e 6 resume os modelos obtidos e a confiabilidade das correlações através dos valores de $\mathrm{R}^{2}$ e Pvalor. Todas expressões obtiveram modelos matemáticos representados por regressão linear simples.

Tabela 5: Modelos de correlação entre parâmetros de Velocidade (V) obtidos por ultrassom, e resistência à compressão (fc), resistência à compressão diametral (ft,D) e módulo de

Elasticidade inicial (Eci), obtidos em ensaio de compressão estática, para amostras sem fibras e com adição de fibras, para as frequência de 45 e $80 \mathrm{kHz}$.

\begin{tabular}{|c|c|c|c|c|c|}
\hline Parâmetro & \multicolumn{2}{|c|}{$\begin{array}{c}\text { Amostra } \\
\text { Frequência do } \\
\text { transdutor }(\mathrm{kHz})\end{array}$} & Modelo & P-Valor & $\begin{array}{l}\mathrm{R}^{2} \\
(\%)\end{array}$ \\
\hline \multirow{2}{*}{ fc } & \multirow{6}{*}{ CPSF } & 45 & $f c=-46,35+0,019 . V$ & 0,0036 & 90,5 \\
\hline & & 80 & $f c=-48,55+0,019 . V$ & 0,045 & 67,5 \\
\hline \multirow{2}{*}{$\mathrm{ft}, \mathrm{D}$} & & 45 & $\mathrm{ft}, \mathrm{D}=-11,30+0,005 \cdot \mathrm{V}$ & 0,0011 & 94,5 \\
\hline & & 80 & $\mathrm{ft}, \mathrm{D}=-14,16+0,006 . \mathrm{V}$ & 0,0052 & 89 \\
\hline \multirow{2}{*}{ Eci } & & 45 & Eci $=-16,16+0,011 . V$ & 0,0041 & 89,75 \\
\hline & & 80 & $\mathrm{Eci}=-17,25+0,012 . \mathrm{V}$ & 0,049 & 67 \\
\hline \multirow{2}{*}{ fc } & \multirow{6}{*}{ CPFA } & 45 & $\mathrm{fc}=-94,93+0,034 . \mathrm{V}$ & 0,0228 & 76,5 \\
\hline & & 80 & $f c=-60,22+0,023 . V$ & 0,0001 & 98,5 \\
\hline \multirow{2}{*}{$\mathrm{ft}, \mathrm{D}$} & & 45 & $\mathrm{ft}, \mathrm{D}=-67,60+0,022 . \mathrm{V}$ & 0,0033 & 91 \\
\hline & & 80 & $\mathrm{ft}, \mathrm{D}=-63,45+0,020 . \mathrm{V}$ & 0,0000 & 99 \\
\hline \multirow{2}{*}{ Eci } & & 45 & $\mathrm{Eci}=-42,26+0,019 . \mathrm{V}$ & 0,020 & 78 \\
\hline & & 80 & $\mathrm{Eci}=-32,53+0,016 . \mathrm{V}$ & 0,0420 & 68,5 \\
\hline \multirow{2}{*}{$\mathrm{Fc}$} & \multirow{6}{*}{ CPFB } & 45 & $f c=-74,30+0,027 . V$ & 0,0018 & 93,5 \\
\hline & & 80 & $f c=-21,33+0,011 . V$ & 0,0028 & 91,5 \\
\hline \multirow{2}{*}{$\mathrm{ft}, \mathrm{D}$} & & 45 & $\mathrm{ft}, \mathrm{D}=-62,80+0,021 . \mathrm{V}$ & 0,0002 & 98 \\
\hline & & 80 & $\mathrm{fb}=-20,23+0,008 . \mathrm{V}$ & 0,011 & 84 \\
\hline \multirow{2}{*}{ Eci } & & 45 & $\mathrm{Eci}=-36,45+0,017 . \mathrm{V}$ & 0,0015 & 94,0 \\
\hline & & 80 & Eci $=-3,15+0,0068 . V$ & 0,0029 & 92 \\
\hline \multirow{2}{*}{ fc } & \multirow{6}{*}{ CPFP } & 45 & $f c=-84,05+0,031 . V$ & 0,0012 & 94,5 \\
\hline & & 80 & $f c=-32,92+0,015 . V$ & 0,0154 & 80,5 \\
\hline \multirow{2}{*}{$\mathrm{ft}, \mathrm{D}$} & & 45 & $\mathrm{ft}, \mathrm{D}=-49,10+0,017 . \mathrm{V}$ & 0,0032 & 91 \\
\hline & & 80 & $\mathrm{ft}, \mathrm{D}=-13,60+0,006 . \mathrm{V}$ & 0,0336 & 72 \\
\hline \multirow{2}{*}{ Eci } & & 45 & Eci $=-40,21+0,018 . V$ & 0,0009 & 95,20 \\
\hline & & 80 & Eci $=-9,54+0,0090 . V$ & 0,0128 & 82 \\
\hline
\end{tabular}

As correlações entre os parâmetros obtidas através de ensaios destrutivos (fc) e (Eci) 
com os parâmetros (V) e (Ed) por propagação de ondas, encontrados nas Tabela 5 e 6, estão dentro das variações estatísticas de modelos encontradas na literatura por Evangelista (2002); (2006); Benaicha et al. (2007); Machado et al. (2009); Trtnik et al. (2009) e Giacon (2010).

De acordo com Evangelista (2002), Bungey e Millard (2006), os erros de predição utilizando ensaios de ultrassom, podem variar de 10 a $30 \%$ quando baseados em ensaios estáticos de compressão em corpos de prova cilíndricos aos 28 dias.

Tabela 6: Modelos de correlação entre módulo de elasticidade inicial (Eci), obtidos em ensaio de compressão estática, e módulo de elasticidade dinâmico obtidos por ensaio de propagação de ondas, para amostras sem fibras e com adição de fibras, para as frequência de 45 e $80 \mathrm{kHz}$.

\begin{tabular}{c|c|c|l|c|c}
\hline \multirow{2}{*}{ Parâmetro } & \multicolumn{2}{|c|}{$\begin{array}{c}\text { Amostra } \\
\text { Frequência } \\
\text { transdutor }(\mathrm{kHz})\end{array}$} & \multicolumn{1}{|c|}{ Modelo } & P-Valor & $\begin{array}{c}\mathrm{R}^{2} \\
(\%)\end{array}$ \\
\hline \multirow{2}{*}{ Eci } & \multirow{2}{*}{$\mathrm{CPSF}$} & 45 & Eci $=3,23+0,65 . \mathrm{Ed}_{45}$ & 0,011 & 83 \\
\cline { 3 - 6 } & & 80 & $\mathrm{Eci}=0,25+0,75 . \mathrm{Ed}_{80}$ & 0,046 & 67 \\
\hline \multirow{2}{*}{ Eci } & \multirow{2}{*}{$\mathrm{CPFA}$} & 45 & $\mathrm{Eci}=3,23+0,70 . \mathrm{Ed}_{45}$ & 0,016 & 80 \\
\cline { 3 - 6 } & & 80 & $\mathrm{Eci}=0,65+0,76 . \mathrm{Ed}_{80}$ & 0,039 & 70 \\
\hline \multirow{2}{*}{ Eci } & \multirow{2}{*}{$\mathrm{CPFB}$} & 45 & $\mathrm{Eci}=-13,20+1,26 . \mathrm{Ed}_{45}$ & 0,0006 & 96 \\
\cline { 3 - 6 } & & 80 & $\mathrm{Eci}=9,70+0,39 . \mathrm{Ed}_{80}$ & 0,0014 & 94 \\
\hline \multirow{2}{*}{ Eci } & \multirow{2}{*}{$\mathrm{CPFP}$} & 45 & $\mathrm{Eci}=-7,1+1,07 . \mathrm{Ed}_{45}$ & 0,0030 & 91 \\
\cline { 3 - 6 } & & 80 & $\mathrm{Eci}=6,77+0,53 . \mathrm{Ed}_{80}$ & 0,011 & 84 \\
\hline
\end{tabular}

Os valores determinados (Ed) para as amostras em estudo superaram em $30 \%$ os valores de (Eci), esta porcentagem esta dentro da margem dos valores encontradas nos estudos experimentais realizados por Metha e Monteiro (2008), Almeida e Hanai (2008) em corpos de prova cilíndricos ensaiados aos 28 dias. Os pesquisadores mostraram que o módulo dinâmico é maior que o módulo estático em torno de $28 \%$ a $40 \%$ para concretos considerados de baixa resistência, com fck $\leq 20 \mathrm{MPa}$, conforme características das amostras em estudo.

Segundo o ACI 228.1R (2003), a utilização de correlações para estimar a resistência do concreto através de propagação de ondas, deve ficar restrita a amostras com traços de mesmas caracterísitcas. As correlações obtidas na presente pesquisa não possuem discrepâncias no comportamento baseado nos valores de $\mathrm{R}^{2}$, quando comparado as modelos para inferir (fc) e (Eci) obtidos através de propagação de ondas encontrados na literatura. As equações apresentadas na Tabela 5 e 6 , podem ser comparadas com os modelos encontrados por Câmara (2006), Machado et al. (2009) e Trtnik et al. (2009), podendo ser utilizadas para a inferência de resistência à compressão simples para concretos entre 5 a $40 \mathrm{Mpa}$. Os modelos obtidos para determinar a resistência à tração são semelhantes ao encontrado por Benaicha et al. (2007) para concretos entre 8 a $25 \mathrm{MPa}$. As correlações para inferir o módulo de elasticidade do concreto possuem mesma faixa de valores $\mathrm{R}^{2}$, encontradas por Rodrigues e Figueiredo (2003) e Prado (2006), Giacon (2010) para módulo de elasticidade entre 15 a 37 GPa. 
Através da análise variância pelo método ANOVA, para os modelos encontrados nas tabelas 5 e 6, com P-Valor menor que 0,05, demonstram existir uma relação estatisticamente significativa entre os parâmetros obtidos por ensaios destrutivos e velocidade de ultrassom, indicando nível de confiança de $95,0 \%$. Os resultados obtidos indicam que a técnica de propagação de ondas de ultrassom é sensível ao uso de fibras no concreto, podendo ser utilizada como solução de ensaio não destrutivo para estimar a qualidade ou, em alguns casos, a resistência do material. Pode-se utiliar as formulações encontradas, desde que estejam dentro da faixa dos valores encontrado pelos ensaios destrutivo e possua o mesmo teor de fibras abordado na pesquisa nos traços de concreto.

\section{CONCLUSÃO}

Através da análise dos resultados obtidos nos ensaios realizados e comparando com estudos anteriores pode-se observar que as fibras de aço atuam de maneira satisfatória quando se deseja aumentar a resistência à compressão e tração do concreto.

Algumas características das fibras podem influenciar diretamente nos resultados dos ensaios como peso, formato e comprimento. As fibras possuem diferentes pesos e resistências individuais, o que altera o volume de fibras na mistura. Nesse caso, há influência sobre a resistência do concreto devido àincorporação de ar na mistura, o que cria pontos de instabilidade na matriz de concreto.

Quanto ao módulo de elasticidade, notou-se que houve uma diferença baixa em relação ao traço utilizado como base para os estudos, sendo que o traço com adição de borracha ainda obteve um desempenho inferior aos demais traços estudados devido a sua baixa aderência com a mistura do concreto, ponto já fixado como uma das principais causas de perda de resistência e módulo de elasticidade.

A propagação de ondas de ultrassom nas amostras não foram afetadas pelas diferentes frequência dos transdutores 45 e $80 \mathrm{kHz}$ e pelos tipos de fibras adicionados na matriz, não influenciando a velocidade de ultrassom para teores de fibra em $2 \%$ no traço de concreto.

As análises estatísticas indicam correlações significativas entre velocidade de ultrassom com resistência à compressão, resistência à tração e módulo de elasticidade, os modelos propostos obtiveram coeficiente de determinação $\left(R^{2}\right)$ bastante elevado, independente do tipo de fibra.

A utilização do método ultrassônico para a inferência da resistência e módulo de deformação estático do concreto é importante para a comunidade técnica e científica, pois trata-se de ensaio de baixo custo e de fácil e rápida execução. Sendo assim, este parâmetro tem grande relevância para a análise da manutenção e do desempenho das estruturas de concreto com moderada adição de fibras. 


\section{REFERÊNCIAS}

ACEBES, M., MOLEERO, M., SEGURA, I., MORAGUES, A., HERNÁNDEZ, M. G. (2011). Study of the influence of microstructural parameters on the ultrasonic velocity in steel-fiber-reinforced cementitious materials. Construction and Building Materials, 25(7), 3066-3072.

AKASAKI, J.L.; FIORITI, C.F.; NIRCHL, G.C. Análise experimental da resistência à compressão do concreto com adição de fibras de borracha vulcanizada. In: $43^{\circ}$ Congresso Brasileiro do Concreto. 2001.

ALBUQUERQUE, A.C.; ANDRADE, M.A.S.; NETO, M.M.S.; CORREA, M.I.F.; CARDOSO, C.G.; MACEDO, D.C.B.; CALMON, J.L. Concreto com borracha de pneu: uma revisão bibliográfica. $43^{\circ}$ Congresso Brasileiro de Concreto - IBRACON. Belo Horizonte, MG. 2002, IBRACON.

ALBUQUERQUE, A.C.; HASPARYK, N.P.; ANDRADE, M.A.S.; CORREA, M.I.F.; NETO, M.M.S.; MACEDO, D.C.B.; CARDOSO, C. G. Investigation of different treatments in tire rubber with a view to concrete application. Conference on use of the recycled materials in building and structures. RILEM. Barcelona, 2004.

ALBUQUERQUE, A.C.; HASPARYK, N.P.; ANDRADE, M.A.S.; ANDRADE, W.P. Polymeric admixtures as bonding agent between tire rubber and concrete matrix. ACI American Concrete Institute. Michigan, Estados Unidos. 2005.

ALBUQUERQUE, A.C. Estudos das propriedades do concreto massa com adição de partículas de borracha. Dissertação (Doutorado em Engenharia) - Universidade Federal do Rio Grande do Sul UFRS. Porto Alegre, RS. 2009.

ALMEIDA, S. F.; HANAI, J. B. Análise dinâmica experimental da rigidez de elementos de concreto submetidos à danificação progressiva até a ruptura. Cadernos de Engenharia de Estruturas, São Carlos, v.10, n. 44, p. 49-66, 2008.

AMERICAN CONCRET INSTITUTE, ACI 228.1R-03. "In-Place Methods to Estimate Concrete Strength", Detroit. 2003.

AMERICAN SOCIETY FOR TESTING AND MATERIALS. ASTM C597-02: standard testmethod for pulse velocity through concrete. Filadélfia, 2002.

ASSOCIAÇÃO BRASILEIRA DE NORMAS TÉCNICAS-ABNT. NBR NM 67. Concreto: determinação da consistência pelo abatimento do tronco de cone. Rio de Janeiro, 1998.

. NBR 9778: Argamassa e concreto endurecidos - Determinação da absorção de água por imersão - Índice de vazios e massa específica. Rio de Janeiro, 2005.

NBR 7222. Argamassa e concreto - Determinação da resistência à tração por compressão diametral de corpos-de-prova cilíndricos. Rio de Janeiro, 2011. 
NBR 16097. Solo - Determinação do teor de umidade - Métodos expeditos de ensaio. Rio de Janeiro, 2012.

NBR 8802. Concreto Endurecido - Determinação da velocidade de propagação da onda ultra-sônica. Rio de Janeiro, 2013.

NBR 5738. Concreto - Procedimento para moldagem e cura de corpos-deprova. Rio de Janeiro, 2016.

. NBR 8522 - Concreto - Determinação dos módulos estáticos de elasticidade e de deformação a compressão. Rio de Janeiro, 2017.

NBR 5739. Ensaio de compressão de corpos-de-prova cilíndricos de concreto. Rio de Janeiro. 2018.

BALENDRAN, R. V., ZHOU, F. P., NADEEM, A., LEUNG, A. Y. T. Influence of steel fibres on strength and ductility of normal and lightweight high strength concrete. Building and environment, v. 37, n. 12, p. 1361-1367, 2002.

BATTAGIN, I.L.S. Módulo de Elasticidade do concreto: como analisar e especificar. Comitê Brasileiro de Cimento, Concreto e Agregados da ABNT. São Paulo, SP. 2007.

BENAICHA, M., JAULBALD, O., AlAOUI, A. H., BURTSCHELL, Y. Correlation between the mechanical behavior and the ultrasonic velocity of fiber-reinforced concrete. Construction and Building Materials, v. 101, p. 702-709, 2015.

BENTUR, A.; MINDESS, S. Fiber reinforced cementitious on durability of concrete. Barking: Elsevier, 1990.

BENTUR, A.; MINDESS, S. Fibre reinforced cementitious composites. 2nd ed. London: Taylor \& Francis, 2007. 625 p.

BOSCO, V. I. D. Análise Comparativa entre Concreto Convencional Reforçado com Fibras de Aço e Polipropileno Através de Ensaio com Propagação de Ondas Ultrassônicas. In: XXVII São de Iniciação Científica, 2015, Universidade Federal do Rio Grande do Sul - Rio Grande do Sul, 2015.

BRITISH STANDARD INSTITUTION. BS 1881:203. Testing Concrete. Recommendations for measurement of velocity of ultrasonic pulses in concrete, London, 1986.

BUCUR, V. Acoustics of wood. 2. ed. New York: Springer-Verlag, 2006, 393p.

BUNGEY, J. H., MILLARD, S. G. GRANTHAM, M. G. Testing of concrete in structures. 4 ed. New York, USA: Champan e Hall, 2006. 310p.

CÂMARA, E. Avaliação da resistência à compressão do concreto utilizado usualmente na grande Florianópolis através de métodos de ensaios não destrutivos. 152f. Dissertação (Mestrado Engenharia Civil) - Universidade Federal de Santa Catarina,Florianópolis, 2006. 
CHUN, Y.; CLAISSE, P.; NAIK, T.R.; GANJIAN, E. Sustainable Construction Materials and Technologies. p. $87-1^{a}$ Edição, Taylor \& Francis. Milton Park, Osfordshire, Reino Unido. 2007.

CIMOLIN, F.R.; GODINHO, D.S.S. Estudo do efeito da fibra de polipropileno em concreto exposto a altas temperaturas. Universidade do Extremo Sul Catarinense UNESC. Criciúma, SC. 2015.

DOMAGAŁA, L. Modification of properties of structural lightweight concrete with steel fibres. Journal of Civil Engineering and Management, v. 17, n. 1, p. 36-44, 2011.

EHRENBRING, H. Z.; TUTIKIAN, B. F.; MEDEIROS, U. C. Q. Análise comparativa da retração por secagem de concretos com fibras novas e recicladas de poliéster. Ambiente Construído, v. 18, n. 3, p. 195-209, 2018.

EVANGELISTA, A. C. J. Avaliação da resistência do concreto usando diferentes ensaios não destrutivos. Tese de Doutorado. UNIVERSIDADE FEDERAL DO RIO DE JANEIRO. 2002.

FIGUEIREDO, A.D. Concreto projetado: Fatores intervenientes no controle da qualidade do processo. Dissertação de mestrado - Escola Politécnica da Universidade de São Paulo - Departamento de Engenharia de Construção Civil. São Paulo, SP. 1992.

FIGUEIREDO, A. D. Concreto reforçado com fibras. Tese Livre Docência. Universidade de São Paulo. 2011.

GENCEL, O.; OZEL, C.; BROSTOW, W.; MARTINEZ, B. Mechanical properties of selfcompacting concrete reinforced with polypropylene fibres. Materials Research Innovations, v. 15, n. 3, p. 216-225, 2011.

GIACON Jr, M., GONCALVES, R., SORIANO, J., AMALFI, G. . Caracterização do concreto utilizando ultrassom. In: XXVIII CONAENDI - Congresso de Ensaios Não Desrutivos e Inspeção, 2010, Santos - SP. Anais CONAENDI 2010, 2010. v. 1. p. 1-9.

GOES, C. M. W.; FONTANI, P. S. P.; PIMENTEL, L. L.; AVILA, A. E. P. G. Análise da aplicaçao de concreto com adiçã de macrofibras estruturasis sintéticas e microfibras de vidreo anti-crack ar e pisos. REEC-Revista Eletrônica de Engenharia Civil, v. 12, n. 1, 2016.

HERSCOVICI, H. L.; ROEHL, D.; SÁNCHEZ FILHO, E. de S. Estudo experimental de vigas curtas de concreto com fibras de aço sujeitas à flexão. Revista IBRACON de Estruturas e Materiais, v. 12, n. 2, p. 288-307, 2019.

LAU, A.; ANSON, M. Effect of high temperatures on high performance steel fibre reinforced concrete. Cement and Concrete Research, v. 36, n. 9, p. 1698-1707, 2006.

LUCENA, J.C.T. Concreto reforçado com fibras de polipropileno: estudo de caso para aplicação em painel alveolar de parede fina. Dissertação (Mestrado em Engenharia de 
Estruturas) - Universidade de São Paulo USP - Escola de Engenharia de São Carlos. São Carlos, SP. 2017.

MACHADO, M. D.; SHEHATA, L. D.; SHEHATA, I. A. E. M. Curvas de correlação para caracterizar concretos usados no Rio de Janeiro por meio de ensaios não destrutivos. RIEM-IBRACON Structures and Materials Journal, v. 2, n. 2, 2009.

MEDEIROS, A. Estudo do comportamento à fadiga em compressão do concreto com fibras. Tese de Doutorado. Pontifícia Universidade Católica do Rio de janeiro. P.201. 2012.

METHA, P.; MONTEIRO, P. Concreto - Microestrutura, Propriedades e Materiais. $3^{\circ}$ edição, Ed Ibracon. 2008.

MOHAMMED, T. U.; RAHMAN, M. N. Effect of types of aggregate and sand-toaggregate volume ratio on UPV in concrete. Construction and Building Materials, v. 125, p. 832-841, 2016.

NOGUEIRA, C.L.; WILLIAN, K. J. "Ultrasonic testing of damage in concreteunder uniaxial compression", ACI Materials Journal, May.-June., pp. 265-275. 2001.

OHDAIRA, E.; MASUZAWA, N. "Water content its effec on ultrasound propagation in concrete - the possibility of NDE", Ultrasonics, 38., pp. 546-552. 2000.

OLIVEIRA, N.P. Concreto de cimento Portland reforçado com fibras de aço e de polipropileno. Curso de Engenharia Civil - Centro Universitário de Formiga UNIFOR. Formiga, MG, 2014.

PILLAR, N. M. P. Propriedades Mecânicas nas primeiras idades como preditoras das Tensões Induzidas e Fissuração de Concreto Projetado Reforçado com Fibras, Tese de Doutorado, Universidade Federal de Santa Catarina, Florianópolis, 2014.

POPOVICS, S. Analysis of the concrete strength versus ultrasonic pulse velocity relationship. Materials Evaluation(USA), v. 59, n. 2, p. 123-124, 2001.

PRADO, L.A. Módulo de deformação estático do concreto de Baixa e alta relação a/c pelo método ultra-sônico. Dissertação (Mestrado em Engenharia Civil) - Escola de Engenharia Civil Universidade Federal de Goiás, Goiânia, 226p. 2006.

RICHARDSON, A. E. Compressive strength of concrete with polypropylene fibre additions. Structural survey, v. 24, n. 2, p. 138-153, 2006.

RODRIGUES, G. S. S.; FIGUEIREDO, E. I. P. Módulo de deformação estático do concreto pelo método ultrassônico: Estudo da correlação. In: $46^{\circ}$ Congresso Brasileiro do Concreto, Florianópolis, 2004. Anais. IBRACON, Florianópolis, p.1309-1324. 2004.

ROSA FILHO, C. D. D.; SILVA, A. J. C. E.; PARISI, S. A.;MONTEIRO, E. C.; ALMEIDA, D. C. Análise do comportamento mecânico do concreto com adição de fibras de polipropileno e sua atuação no combate às manifestações patológicas. In: Anais da 
Conferência Nacional de Patologia e Recuperação de Estruturas. 2017.

SEGRE, N. Reutilização de borrachas de pneus usados como adição em pasta de cimento. Tese de doutorado - Universidade Estadual de Campinas UNICAMP. Campinas, SP. 1999.

SEGRE N.; MONTEIRO, P.J.M.; SPOSITO, G. Surface characterization of recycled tire rubber to be used in cement paste matrix. Journal of Colloid and Interface Science, Vol. 248. Rússia, 2002.

SILVA, P. M. Análise da perda de rigidez em vigas de concreto armado devido à fissuração por esforços de flexão utilizando ensaio de ultrassom. Universidade Federal de Santa Catarina. Dissertação de Mestrado. p.159. 2017.

STURRUP, V. R., Vecchio, F. J., and Caratin, H. "Pulse Velocity as a Mesuare of Concrete Compressive Strength", In Situ/Non destructive Testing of Concrete, Special Publication SP-82, American Concrete Institute, Detroit, pp.201-228. 1984.

TRTNIK, G.; KAVČIČ, F.; TURK, G. Prediction of concrete strength using ultrasonic pulse velocity and artificial neural networks. Ultrasonics, v. 49, n. 1, p. 53-60, 2009.

YU, T.; CHAIX, J. F.; AUDIBERT, L.; KOMATITSCH, D.; GARNIER, V.; HENAULT, J. $M$. Simulations of propagation of ultrasonic waves in concrete based on a twodimensional numerical model validated analytically and experimentally. Ultrasonics, 92, 21-34. (2019). 